\title{
Lora System based Accident Rescue System for Future Generation
}

\author{
${ }^{1}$ N.V.K. Ramesh, ${ }^{1}$ Puctchapurnapriya, ${ }^{1}$ N. Suresh, ${ }^{2}$ Shaik Razia, ${ }^{3}$ B Naresh Kumar Reddy, ${ }^{4}$ P. Gopi Krishna \\ ${ }^{1}$ Department of ECE, Koneru Lakshmaiah Education Foundation, Vaddeswaram, Andhra Pradesh, India, nvkr@kluniversity.in, \\ putchapoornapriya@kluniversity.in, namgirisuresh@kluniversity.in \\ ${ }^{2}$ Department of CSE, Koneru Lakshmaiah Education Foundation, Vaddeswaram, Andhra Pradesh, India, skrazia@kluniversity.in \\ ${ }^{3}$ Department of ECE, ICFAI-TECH, Hyderabad, Telangana, India, naresh.nitg@ gmail.com \\ ${ }^{4}$ Department of ECM, Koneru Lakshmaiah Education Foundation, Vaddeswaram, India, gopikrishna.popuri@gmail.com
}

\begin{abstract}
Fatal crashes are increasing exponentially in India. Death rates have a high impact on any Nation. The existence of the harmed individual could be spared by providing clinical assistance at the right time. The conventional techniques are basically focused on transmitting messages using the cell arrangement. This endeavour's technique is to recognize the area of the accident region and send those directions to the nearest locations of clinical assistance. With the aid of the Bluetooth gadget the communication occur between the commuter and the device. GPS gadget is very noteworthy for the recognizable proof of the present location. Using GPS this will eliminate the directions of the accident prone zone. The geographic directions of the different medical clinics will be stored in the database. It is best to achieve the directions of the accident prone region and medical clinic directions in the database, as well as to determine the distance between them. The directions of the closest available medical clinics can be seen and communication can be established with the health facility.
\end{abstract}

Key words: GPS, Wireless control, FEC, CSS, SP

\section{INTRODUCTION}

The automotive industry occupies an extraordinarily significant role in the society. Our existence is totally dependent upon these automakers. In our society, one lakh fifty thousand people are murdered in mishaps per annum. It's incredibly challenging to secure an individual's existence when he's faced with a mishap when he can't get any assistance. In India [1] individual kick a bucket at regular intervals due to mishap. Road safety and security is a big problem in India. Numerous lives have been destroyed because of street mishaps. As per the survey released by the WHO, one lakh citizen was promptly moved to the mishap area in 2013.The fatal crash risk can be diminished by hitting one of the closer clinical locations. The main focus of the aspect presented in this paper is the delivery of messages to the nearest emergency aid areas. Global Positioning System is a piece of software utilized for the following locations. This appliance defines the region by geostationary correspondence. In any case, it gathers information via 3-4 satellites and determines respective regions. This gadget uses the Triangulation technique to define the zones, and range computing becomes extremely easier afterwards. The Bluetooth module is a portion of this framework. The Bluetooth setup is used for quick distance correspondence. The classical mishap framework isn't really worried regarding the condition of the motorist. The device uses a $2.45 \mathrm{GHz}$ radio recurrence for correspondence. Standard frameworks use GSM for the transmission of messages. [2] This paper presents our analysis taking a shot at the execution of LORA idea in the position of mishap and ensuring the health of the rider. This framework uses LORA innovation to convey the instructions of the collision zone to the nearby clinics, so that timely assistance can be sent by the clinic to the place where the mishap has occurred. LORA is a gadget focussed upon semtech's restrictive range change strategy. LORA Device is a mixture of CSS (Chirp Spread System) and FEC (Forward Error Correction).LORA devices work among both $0.137 \mathrm{GHz}$ to $1.020 \mathrm{GHz}$. Accordingly this device will work in certified and certified free of charge. Lora follows the Structure of STAR network. Star topography is a protocol where aamount of $s$ is correlated with a central storage. It responds to the customer in the light of worker accessibility. The LORA system described for this framework is LORASX1278 Ra-02 that functions at $0.433 \mathrm{GHz}$. The key reason with this article is to suggest a solution that can fix the existing problem.

\section{LITERATURE SURVEY}

A great deal of exploration is being carried in the field of mishap location and recuperation. JubayerJalil et al presented a system that would rely on the velocity. This complete circuit uses the ability of the Global Positioning System and the accelerometer to track the area and travelling velocity. At a point where the downward that speeds reaches the limit value, the explanation for this is that the vehicle is having a mishap. The framework uses the Global System for Mobile Communication to pass the mishap region to the nearby emergency aid zones. [3] The final part of the system consists of above discussed devices to transfer the region of the accident 
N.V.K. Ramesh et al., International Journal of Emerging Trends in Engineering Research, 8(9), September 2020, $5022-5027$

zone to the nearest clinical focal point. The big downside to this methodology is that the mechanism is never sensitive to the motorist's condition. No need to decrease the speed immediately after the mishap has happened. Shaik Mazhar Hussain et al proposed the process to minimize each danger posed by mishaps. This idea is entirely focused on the application of IR sensors. The approach employed in this framework consists of two phases. The primary stage is the recognition of mishap, and the next stage focuses on diagnosing mishap. The main period of the device includes infrared alert sensors at any point where the distance among any different vehicle becomes smaller than the permissible limit. The suggested model could not be implemented in regions with significant congestion. The response to the question was followed by Nagendra Kumar et al. The structure is to organise information on the vast number of sensing devices used by the framework. This element proposes that the mishap could be detected through the use of sensors. [4] The method also incorporates the versatility of the portable sensing devices. The wellbeing of the motorist could be resolved with the assistance of the portable sensing devices. At a time when a motorist is experiencing a mishap, his well-being can be fixed. The exactness of portable sensing devices is significant in order to assess the wellbeing circumstances. The framework proposed throughout the article utilizes LORA innovation for communicating the directions of mishap zone towards nearby emergency clinics

\section{DESIGN METHODOLOGY}

The basic purpose of this endeavour is to provide the victim's life during a mishap. Bluetooth 5.0 is a technology that is utilized for smaller connectivity. At the point where a vehicle is struck by a mishap, a notification was sent through frame to the motorist's enrolled contact. [5] This framework holds fast for duration of forty five sec. Also, this framework concludes that sending the area of the mishap vehicle to the emergency clinics and the police headquarters would rely upon this motorist's response.

\subsection{Approach for identifying the surrounding healthcare facilities}

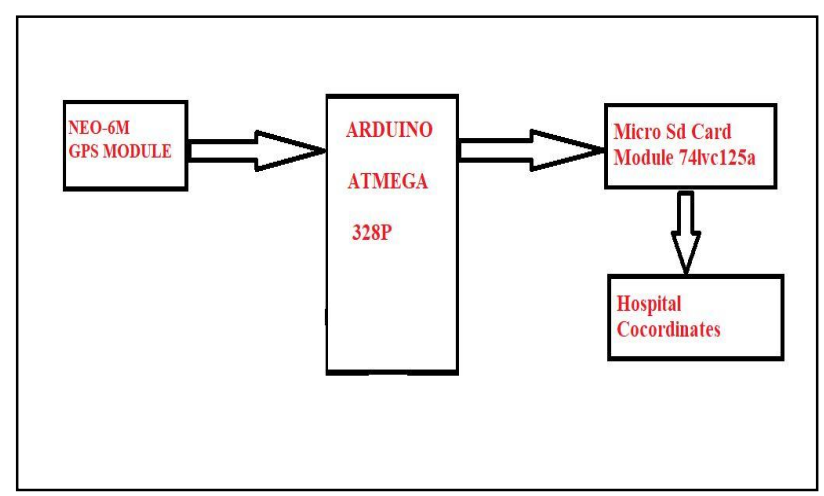

Figure 1: Circuit design for locating healthcare facilities
The layout in Figure 1 illustrates the concept of exploring the distance and longitude of the nearest available medical clinics. [28] The region characterised by the use of navigation system is the contribution to Microcontroller. [6] The zone is identified by the GPS is an engagement with the Arduino Atmega 328P.

Distance $=\operatorname{acos} \quad(\sin \quad($ latitude1) $* \sin \quad$ (latitude2) $+\cos$ (latitude1)*cos (latitude2)*cos (longitude1-longitude2) Longitude 1 is the longitude estimation of the GPS arranges and longitude 2 is the longitude estimation of the emergency clinics put away in the server base.[27] The locations of the minimum distinction will just be retained and the areas enclosed by the GPS shall be sent to the respected directions. Latitude1 is the estimate of the scope of the GPS facilitates and Latitude 2 is the estimate of the scope of the clinics in the server base.

\subsection{Expected structure}

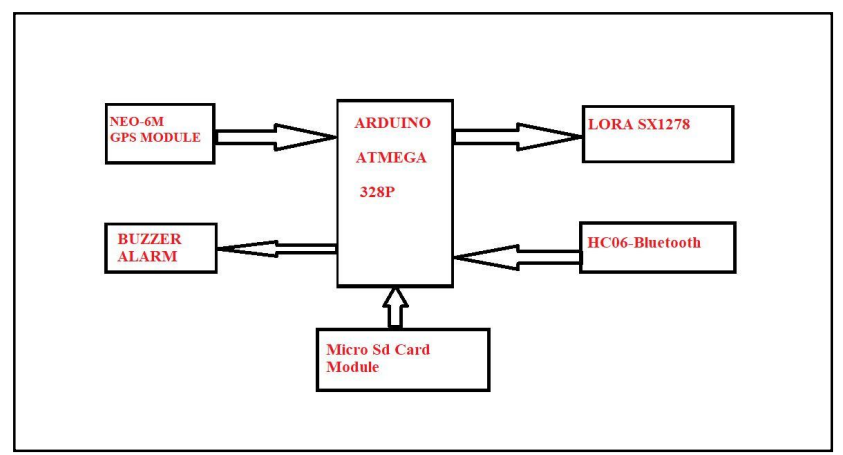

Figure 2: Circuit diagram of the framework proposed

The framework structure shown in fig 2 shows the technique included in this application. [10] The exact location that suffered a mishap is recognized utilizing GPS technology. We use the Neo-6 m GPS module throughout this design. If the incident happens, the device initiates a call to the motorist's telephone number. The answer time shall be determined. The next step is to have the current location and submit it to the nearby healthcare facility. When contact is underway, the motorist will be made it known with the aid of Buzzer Warning. The position is being sent through log range gateway. The Long Range device shown in this is LORA SX1278.Medical addresses will be kept in a centralized folder. The Micro SD card module (74LVC125A) is often used to save the details of the emergency services. The mechanism used by this device could be Serial Peripheral Interface (SPI).

Any encapsulated architecture shall be centered on Controllers and devices In order to create an inter-process contact among the framework equipment, a widely accepted mechanism for information exchange should be discussed. [7] The existing protocol implements the guidelines of the Serial Peripheral Interface (SPI). There have been 3 ways to communicate:

Simplex: The data sharing occurs in a simplex model only in single manner. It is from the source to the receiver or from the receiver to the source. 
Half-Duplex: The Correspondence signal is communicated in one single direction. The exchange of data normally occurs in the path from source to destination. The data can be shared in both the ways. The data cannot be shared through both channels at the same period of time.

Full-Duplex: Correspondence signal is communicated in one single direction. The exchange of data normally occurs in the path from source to destination, but it is possible to pass through both channels at the same period of time.

The SPI protocol is based on a full-duplex procedure. Thus, information may be sent from Microcontroller to any device and from device to Microcontroller simultaneously. [29], It's a concurrent, simultaneous protocol. This negotiation potential for the Master-Slave union. This procedure is suited for Synchronous transfer. Synchronous transfer implies 1 byte of information is uprooted at 1 clock beat. The whole framework is coordinated with the clock. The transmitter and the receiver clocks work at equal recurrence. Synchronized means of transport set aside less time for removal. [30] The Arduino clock recurrence is $16 \mathrm{MHz}$ and all the devices connected to Arduino do not operate at a similar clock recurrence. The crucial downside to this convention is that as the number to accessories are attached to the microcontroller, the challenges of the framework increase. 8 The amount of pins needed by the Master is dependent on the number of slaves associated with that Microcontroller the total amount of connections needed in the SPI correspondence is: $3+\mathrm{B}$. Here, the B is the number of gadgets tied to a specific Microcontroller.

The following modes are used:

\begin{tabular}{|l|l|l|l|}
\hline \multirow{2}{*}{ SPI Mode } & Clock Polarity & \multicolumn{2}{l|}{ Clock Edge//Phase } \\
\cline { 2 - 4 } & CPOLCKP & CPHA & NCPHA/CKE \\
\hline 0 & 0 & 0 & 1 \\
\hline 1 & 0 & 1 & 0 \\
\hline 2 & 1 & 0 & 1 \\
\hline 3 & 1 & 1 & 0 \\
\hline
\end{tabular}

Figure 3: SPI correspondence Protocol Modes

The table seen in picture 3 shows the 4 modalities of a SPI inter-process communiqué. Whenever the polarity of the clock is zero and the phase of the clock is zero then the instructor transmits the signal to clock signal. Because once phase 1 is finished, the instructor gets data also at output port of the clock. When the polarity of the clock is zero and phase is one then the signal is recorded at the rising edge and data is moved on rising edge of the clock. [9] In the second mode, the signal is registered on the dropping edge of the clock pulse and transferred to the growing edge pulse. In third mode signal is recorded at growing edge and is moved at the dropping edge pulse.

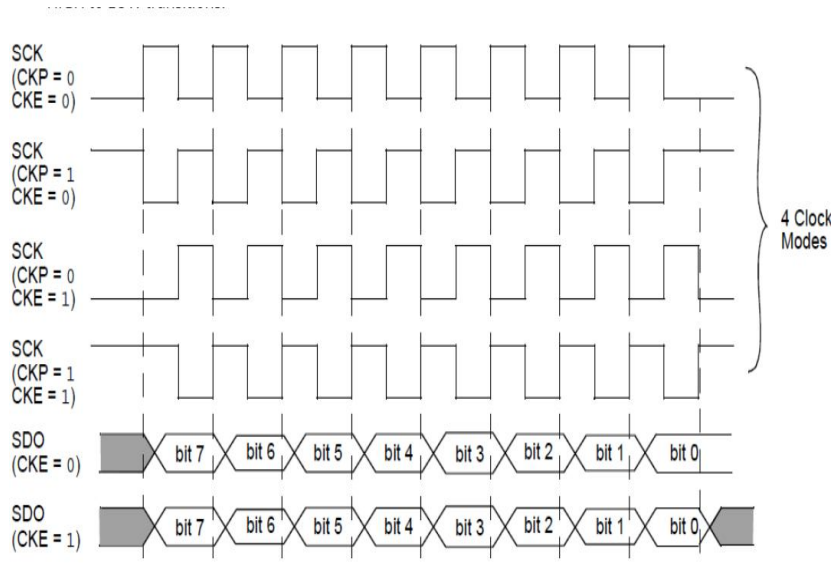

Figure 4: Clock Drawing of SPI Protocol

\subsection{Components}

1) HC-06 Bluetooth Device: The Bluetooth gadget has been used for a smaller interval chart. It is an RF correspondence with streak storage, a Bluetooth base band controller, an RS232 interface and a radio unit. [31] Bluetooth is a remote conveyance technique that operates at $2.45 \mathrm{GHz}$. In our job, we use Bluetooth to handle the circumstances of the motorist after a mishap has occurred. Wherever a mishap is identified, a spurious text is forwarded to a telephone number of the individual. At a point where the motorist responds to it by forty five sec of pause, and thereby ensures that the motorist is in a competent state and can support himself. In the off chance that there is no reaction structure, the motorist agrees that the person is unaware and wants a prompt health assistant. Bluetooth imparts the sign to the regulator to get vigorously.

2)LORA SX 1278 Device: [9] The recurrence frequency of LORA SX1278 is $137 \mathrm{MHz}$ to $525 \mathrm{MHz}$.The cutting edge Long Range module covers a diverse range that allows us to reach high-level correspondence. It eliminates the disruption of noises. Modulation is a method through which researchers include the signal intensity such that the message can be transmitted over lengthy ranges.

3) Neo-6m GPS Device: This device is utilized to identify the source. This hardware is designed using Electrically Erasable Programmable Read Only Memory for redundancy of data. GPS is used throughout the project to know the exact destination where the crash happened. The nominal voltage for this unit is 3.3V. The GPS module is designed between 4800 baud rates to 115200 baud rate. The baud rate of 9600 has been the default baud rate.

4) Micro SD Card Device (74LVC125A): The Micro SD card obeys the procedure of the SPI. The whole procedure has been developed by Motorola. The appliance obeys a full-duplex framework. So, information can be transferred in either manner. The data is moved from instructor to servant and also from servant to instructor at almost the same period. The $74 \mathrm{LVC1} 25 \mathrm{~A}$ appliance is used as a dataset to hold the health facility exact location. 
N.V.K. Ramesh et al., International Journal of Emerging Trends in Engineering Research, 8(9), September 2020, 5022 - 5027

5) Arduino Atmega 328P device: 10The Atmega328P is an electronic device formulated by AVR. The Harvard 8-bit RISC processor has been updated. The microcontroller has been utilized towards the development of this framework. Handling of inputs and other activities shall be in accordance in this device.

\section{RESULTS}

GPS Analysis

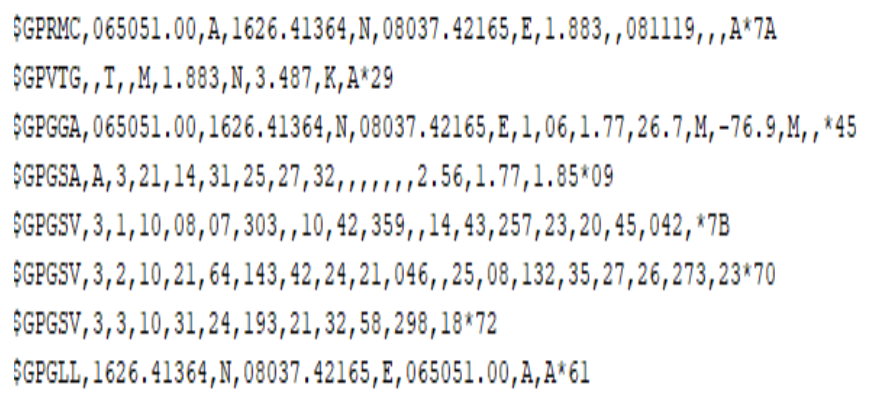

Figure 5: Disaster Zone spot

\section{The shortest health care coordinates}

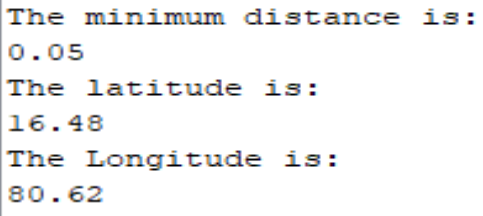

Figure 6: Coordinates of closest Medical Health Centre

\section{Lora Communicator Result}

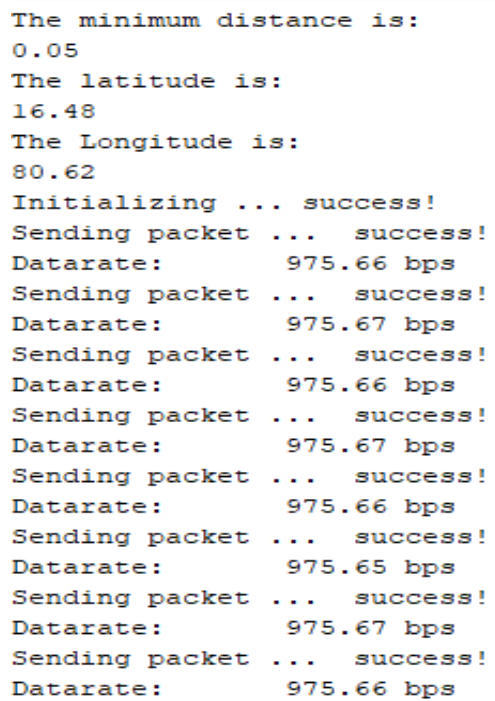

Figure 7: Position communicated with closest medical heath centre

\section{Lora Collector}

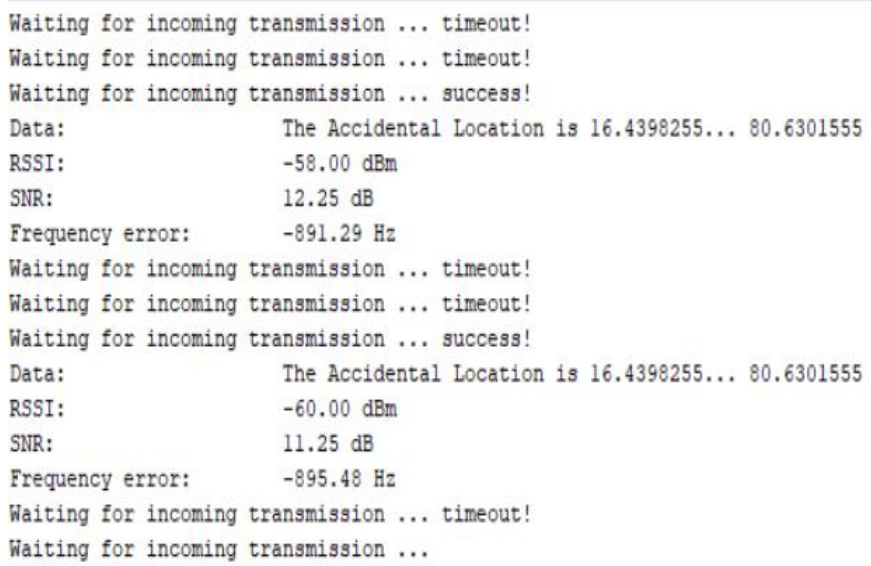

Figure 8: Position received at the closest medical health centre

Figure 5 displays the Global Positioning System's performance whenever connected to Microcontroller. The \$GPGGA is the Generalized Gradient Approximation procedure header. 062828 is a moment when Global Positioning System is attached to the Microcontroller. [11] The number beside the period was the latitudinal points of the position proceeded by direction. The longitudinal point is 08037, accompanied by direction. $\mathrm{N}$ refers to North, e refers to East. 05 represent the amount of satellites used for the position comparison by the Navigation system. Diagram 6 shows the efficiency when Microcontroller was linked to the Repository to obtain those positions that are closest Health Care. Lora's transmission performance is defined in Figure 7 once it is connected to the Microcontroller. Fig 8 indicates results of the receiving Lora Device. The RSSI displays the frequency of the received signal. The signal amplification becomes quite strong when the RSSI value is similar to 0.Signal-to-Noise ratio is the proportion of both the receiver end and the undesirable signals that are introduced into communication channel. Magnitude of Signal-to-Noise ratio should be around $+10 \mathrm{db}$ at the output side to achieve excellence.

\section{CONCLUSION AND FUTURE SCOPE}

In this paper the LORA transmission performance based on Lora sensor is formulated and the frame work activity is effectively tried for a scope is kept intuitive It is best to achieve the directions of the accident-prone region and medical clinic directions in the database, as well as to determine the distance between them. The contrived frame work is effectively versatile. when it is connected to Micro Controller and observed that RSSI displays the frequency of the received signal. When the RSSI value is similar to signal, noise ratio, signal amplification is high. A future work is to be consolidate more sensors it can be extended nearest medical clinics and communication can be established for rural areas in future. 
N.V.K. Ramesh et al., International Journal of Emerging Trends in Engineering Research, 8(9), September 2020, $5022-5027$

\section{REFERENCES}

1. Shaik Ashfaq Hussain and Ajay Vikram Singh "Accidents Detection and Prevention System to reduce traffic Hazards using IR Sensors" in 2018 7th International Conference on Reliability, Infocom Technologies and Optimization (Trends and Future Directions) (ICRITO) . ISBN No: 978-1-5386-4693-9.

2. Md. Syedul Amin, Jubayer Jalil and M. B. I. Reaz "Accident Detection and Reporting System using GPS, GPRS and GSM Technology " in IEEE/OSA/IAPR International Conference on Informatics, Electronics \& Vision. ISBN No: 978-15386-4693-9.

3. Dr.D.Selvathi "Intelligent Transportation System for Accident prevention and detection" in International Conference on Intelligent Computing and Control Systems ICICCS 2017.

4. Venkata Krishna Kota, Nagendra Kumar Mangali, Thirumal Kumar Kanakurthi, A. Rakesh Kumar and and T. Velayutham "Automated Accident Detection and Rescue System" in IEEE Wisp NET 2017 conference.

5. N.V.K Ramesh "Automatic speed controlling of vehicle and detection and notification of path loss and Humps" in Journal of Engineering Applies Science, volume 11 , 2016 ISSN No: 1816-949

6. Dr.N.V.K.Ramesh "Wi-fi controlled universal remote using esp8266" 7233-7237 SSN 1819-6608 December 2017

7. N.V.K.RAMESH, "Development of a smart city using mobile tracking system", in IJAIR. June 2015(72787844) 55 Volume 4 issue 6.

8. Lingling Li, JiuchunRen, Qian Zhu,"On the Application of LoRa LPWAN Technology in Sailing Monitoring System" in 2017 13th Annual Conference on Wireless On-demand Network Systems and Services (WONS) ISBN: 978-3-901882-88-3.

9. Martin Bor, UtzRoedig "LoRa Transmission parameter selection" in 2017 13th International Conference on Distributed Computing in Sensor Systems (DCOSS) ISSN: 2325-2944.

10. Balram G., Kiran Kumar K.(2018), Smart farming: Disease detection in crops', International Journal of Engineering and Technology(UAE),7(2),PP. 33- 36

11. B. Naresh Kumar Reddy, M.H.Vasantha and Y.B.Nithin Kumar, "A Gracefully Degrading and Energy-Efficient Fault Tolerant NoC Using Spare core", 2016 IEEE Computer Society Annual Symposium on VLSI, pp. 146-151, 2016.

12. B.Naresh Kumar Reddy, M.H.Vasantha, Y.B.Nithin Kumar and Dheeraj Sharma, "Communication Energy Constrained Spare Core on NoC", 6th International Conference on Computing, Communication and Networking Technologies (ICCCNT), PP. 1-4, 2015.

13. B. Naresh Kumar Reddy, M.H.Vasantha, Y.B.Nithin Kumar and Dheeraj Sharma, "A Fine Grained Position for Modular Core on NoC IEEE International Conference on Computer, Communication and Control, PP. 1-4, 2015.
14. NKR Becchu et al., "System level fault-tolerance core mapping and FPGA-based verification of NoC", Microelectronics Journal, Vol 70, pp. 16-26, 2017.

15. BNK Reddy et al., "An energy-efficient fault-aware core mapping in mesh-based network on chip systems," Journal of Network and Computer Applications, Vol 105, pp. 79-87, 2017.

16. NKR Beechu et al., "High-performance and energyefficient fault-tolerance core mapping in NoC", Sustainable Computing: Informatics and Systems, Vol 16, pp. 1-10, 2017.

17. BNK Reddy et al., "Energy-Aware and ReliabilityAware Mapping for NoC-Based Architectures," Wireless Personal Communications 100 (2), 213-225, 2018.

18. NKR Beechu, VM Harishchandra, NKY Balachandra, "Hardware implementation of fault tolerance NoC core mapping Telecommunication Systems 68 (4), 621-630, 2018.

19. B. Naresh Kumar Reddy, and B. Sireesha, "An Efficient Core Mapping Algorithm on Network on Chip," 22 nd International Symposium on VLSI Design and Test (VDAT), 2018.

20. NVK Ramesh "Maize Leaf Disease Detection Using Convolution Neural Network", Jour of Adv Research in Dynamical \& Control Systems 12 (02), 1154,2020

21. Shaik Razia "Reducing Overfitting Problem in Machine Learning Using Novel L1/4 Regularization Method", 2020 4th International Conference on Trends in Electronics and Informatics ..., 2020 (IEEE Xplore).

22. Shaik Razia "Fuzzy rule based intelligent system for user authentication based on user behaviour", Journal of Discrete Mathematical Sciences and Cryptography 23 (2), 409-417, 2020.

23. Shaik Razia“A Comparative study of machine learning algorithms on thyroid disease prediction" in Scopus indexed journal (IJETUAE) International Journal of Engineering and Technology(UAE), ISSN No: 2227-524X, Vol No: 7, Issue No: 2.8, Page No: 315-319, March 2018.

24. Shaik Razia“"Development and Analysis of Support Vector Machine Techniques for Early Prediction of Breast Cancer and Thyroid", Journal of Advanced Research in Dynamical and Control Systems, 2017.Vol.9.Sp.Issue:6 ISSN: 1943-023X page no: 869-878).

25. B. Naresh Kumar Reddy, Dharavath Kishan and B. Veena Vani" Performance constrained multiapplication network on chip core mapping," International Journal of Speech Technology, 2019.

26. BNK Reddy, C Ramalingaswamy, R Nagulapalli, D Ramesh, "A novel 8T SRAM with improved cell density", Analog Integrated Circuits and Signal Processing 98 (2), 357-366.

27. S Ahmed, NVK Ramesh, BNK Reddy, "A Highly Secured QoS Aware Routing Algorithm for Software Defined Vehicle Ad-Hoc Networks Using Optimal 
N.V.K. Ramesh et al., International Journal of Emerging Trends in Engineering Research, 8(9), September 2020, $5022-5027$

Trust Management Scheme", Wireless Personal Communications, 1-15,

28. "IoT Based Smart System for Avoidance of Fire Accidents on Running Buses" in International Journal of Engineering \&Technology, 7 (3.12) (2018) 536540.

29. Implementation of Environment Gases Monitoring System using LoRa Gateway in Smart Cities with IoT Technology, P Gopi Krishna, Jour of Adv Research in Dynamical \& Control Systems 12 (02), 1109,2020

30. "GPS and GSM Enabled Embedded Vehicle Speed Limiting Device" in Indian Journal of Science and Technology, Vol 9(17). May 2016.

31. "An IoT based LPG leakage sensing and alerting system" in International Journal of Innovative Technology and Exploring Engineering, (2019) 8(6), pp. 1155-1161.

32. Srinivasa Rao Y., Ravikumar G., Kesava Rao G., Syed M.S. (2017),' Interconnected transmission line fault detection using wavelet transform and a novel machine learning algorithm',Journal of Advanced Research in Dynamical and Control Systems,9(12),PP.142-150.

33. Ayushree; Arora, Sandeep Kumar published "Comparative Analysis Of Aodv And Dsdv Using Machine Learning Approach In Manet", Journal Of Engineering Science And Technology,2017, vol-12, issue 12,pages 3315-3328.

34. Bashir, Ali Kashif, Arul, Rajakumar, Nawab Muhammad Faseeh, published "An Optimal Multitier Resource Allocation Of Cloud RAN In 5G Using Machine Learning" Transactions On Emerging Telecommunications Technologies,2019, VOl30,Issue8, 10.1002/ett.3627

35. Prasad, GSV; Ratnam, DV published, A Deep Learning-Based Approach to Forecast Ionospheric Delays for GPS Signals, IEEE GEOSCIENCE AND REMOTE SENSING LETTERS, 2019,Vol-16,Issue 8, Pages: 1180-1184.

36. Dudi B., Rajesh V. (2019), 'Medicinal plant recognition based on CNN and machine learning', International Journal of Advanced Trends in Computer Science and Engineering, 8(4), PP.9991003 .

37. "An Advanced Crop Field Monitoring System In Agriculture Through Java Beans And Gsm Modem" in International Journal of Recent Technology and Engineering (IJRTE), Volume-8, Issue-1, May 2019.

38. "Super-pixel segmentation and saliency-based plant leaf disease detection using SVM" in International Journal of Recent Technology and Engineering, (2019) 7(6), pp. 710-714.

39. Chowdary T.A., Chakravarthy D.V., Rupesh R.V.S., Ashish T.S.C., Charan V.H.S. (2019), 'Effective implementation of low-cost smart irrigation system', International Journal of Innovative Technology and Exploring Engineering, 8(6), PP.1805-1810.

40. Dukkipati, Sudha; Sankar, V.; Varma, P. Srinivasa, Forecasting of Solar Irradiance using Probability Distributions for a PV System: A Case Study,
INTERNATIONAL JOURNAL OF RENEWABLE ENERGY RESEARCH, 2019, vol-9, issue-2,pages 741-748.

41. Dr.M.Nageswara Rao, Vara Kumari.S Published "Modrak Algorithm to Minimize Completion Time for n-Jobs m-Machines Problem in Flexible Manufacturing System”, ISSN No. 2347 -3983, International Journal of Emerging Trends in Engineering Research, (IJETER) August 2020 , Vol no:8, issue :8, Pages: 4560-4566.

42. Shaik Razia, Venkata Ramani Varanasi published "A Comparative Evaluation of supervised and unsupervised algorithms for Intrusion Detection", International Journal of Advanced Trends in Computer Science and Engineering, (IJATCSE) ISSN 2278-3091, Volume 9 No.4 (2020), pages 48344843,2020 .

43. N V K Ramesh, R A Patil published "Performance Analysis and Improving Connectivity using Quality of Service-aware Routing Algorithm in Vehicular Network" International Journal of Advanced Trends in Computer Science and Engineering, (IJATCSE) ISSN 2278-3091, Volume 9 No.4 (2020), pages 55225528,2020 . 